\title{
Tissue-specific mitochondrial heteroplasmy at position 16,093 within the same individual
}

\author{
Kaarel Krjutškov $\cdot$ Marina Koltšina • \\ Kelli Grand • Urmo Võsa • Martin Sauk • \\ Neeme Tõnisson $\cdot$ Andres Salumets
}

Received: 14 May 2013/Revised: 27 June 2013/ Accepted: 29 June 2013/Published online: 11 July 2013

(C) The Author(s) 2013. This article is published with open access at Springerlink.com

\begin{abstract}
Human mitochondrial DNA (mtDNA) research has entered a massively parallel sequencing (MPS) era, providing deep insight into mtDNA genomics and molecular diagnostics. Analysis can simultaneously include coding and control regions, many samples can be studied in parallel, and even minor heteroplasmic changes can be detected. We investigated heteroplasmy using 16 different tissues from three unrelated males aged 40-54 years at the time of death. mtDNA was enriched using two independent overlapping long-range PCR amplicons and analysed by employing illumina paired-end sequencing. Point mutation heteroplasmy at position $16,093(\mathrm{~m} .16093 \mathrm{~T}>\mathrm{C})$ in the non-coding regulatory region showed great variability among one of the studied individuals; heteroplasmy extended from $5.1 \%$ in red bone marrow to $62.0 \%$ in the bladder. Red $(5.1 \%)$ and yellow bone marrow $(8.9 \%)$
\end{abstract}

Communicated by S. Hohmann.

Electronic supplementary material The online version of this article (doi:10.1007/s00294-013-0398-6) contains supplementary material, which is available to authorized users.

\section{K. Krjutškov $(\varangle) \cdot$ A. Salumets}

Competence Centre on Reproductive Medicine and Biology,

Tartu, Estonia

e-mail: kaarel.krjutshkov@gmail.com

\section{K. Krjutškov · U. Võsa}

Estonian Genome Center, University of Tartu, Tartu, Estonia

K. Krjutškov

Department of Biosciences and Nutrition, Karolinska Institute, Huddinge, Sweden

M. Koltšina · K. Grand · U. Võsa · M. Sauk · N. Tõnisson Department of Biotechnology, University of Tartu, Tartu, Estonia clustered into one group and two arteries and two aortas from different locations into another (31.2-50.9\%), giving an ontogenetic explanation for the formation of somatic mitochondrial heteroplasmy. Our results demonstrate that multi-tissue screening using MPS provides surprising data even when there is a limited number (3) of study subjects and they give reason to speculate that mtDNA heteroplasmic frequency, distribution, and even its possible role in complex diseases or phenotypes seem to be underestimated.

Keywords Human tissue-specific mtDNA heteroplasmy · Illumina sequencing

\section{Background}

Mitochondria that host 37 genes within $16.6 \mathrm{~kb}$ of their genome (mtDNA) are the power engines of the cell. Mitochondrial DNA (mtDNA) gene regulation and replication start from the non-coding control region, D-loop, that is $1,124 \mathrm{bp}$ long from positions $16,024-576$. The

N. Tõnisson

Department of Genetics, United Laboratories, Tartu University Hospital, Tartu, Estonia

A. Salumets

Department of Obstetrics and Gynaecology, University of Tartu, Tartu, Estonia

\section{A. Salumets}

Institute of Bio- and Translational Medicine, University of Tartu, Tartu, Estonia 
D-loop has three hypervariable regions (HVRI-III) and contains $25 \%$ of the variable sites, even though it represents only $7 \%$ of the total mtDNA length (Sosa et al. 2012). Mitochondrial diseases associated with mtDNA mutations and its phenotypic heterogeneity can be explained by mtDNA heteroplasmy-the mixture of more than one type of mtDNA at a cellular, tissue or organism level. The 100-fold higher mutation rate of mtDNA than nuclear DNA is caused by a combination of oxidative microenvironment, lack of histones, and rudimentary repair mechanisms, leading to mtDNA mutation accumulation during aging. Although mtDNA is maternally inherited in mammals, heteroplasmy levels vary markedly between a mother and each of her offspring. This means that asymptomatic mothers with medium to low heteroplasmy levels can have an affected child with much higher levels of the same heteroplasmic mutation ( $>30 \%$ ), which may cause a mitochondrial disease (Zhang et al. 2012).

Massively parallel sequencing (MPS) allows simultaneous analysis of the complete mtDNA and provides annotation and mutant load estimation of each position. Taking into account the variable sequencing depth of published data, small sample sizes, read mapping and data filtering criteria, and heteroplasmy detection thresholds, there is no consensus of opinion as regards average heteroplasmic frequency distribution among individuals and in populations. Full-length mtDNA analysis from 131 individuals identified 40 point heteroplasmies and indels at $10 \%$ minor allele frequency (MAF) or higher in blood or saliva, making 0.3 heteroplasmies per individual ( $\mathrm{Li}$ et al. 2010). In contrast, 1.8 heteroplasmies per individual at $>10 \%$ MAF were identified using 40 immortalized lymphoblastoid cell line samples (Sosa et al. 2012). In recent reports (Andrew et al. 2011; Goto et al. 2011; Avital et al. 2012), variable heteroplasmy in the human body has been demonstrated to exist within a limited number of tissues such as blood, skeletal muscle and buccal epithelium, leaving the rest of the organism out of focus. As multiple tissue analysis in healthy individuals is highly complicated, data on whole body mtDNA heteroplasmy is still far from clear.

In this study, we were able to investigate three unrelated males by employing MPS technology to detect mtDNA heteroplasmy using 16 different tissues taken during post-mortem examination. We show a great range of heteroplasmy variance at position 16,093, where the lowest values were detected in red and yellow bone marrow tissue and the highest in the bladder. The data contribute to mtDNA genetics at human body level and explain the possible formation and expansion of heteroplasmy during post-zygotic embryonic development.

\section{Methods}

The Research Ethics Committee of the University of Tartu approved the collection of tissue samples for research. The written informed consent was obtained from next-of-kin to post-mortem individuals to collect the tissue panel during the autopsy. Encoded body panel was collected from three males of 40 (sample KT538), 53 (KA522) and 54 (SJ600) years of age at the time of death. Donors KT538 and KA522 had died of acute cardiovascular insufficiency due to myocardial infarction and SJ600 of cerebellar haemorrhage. The tissue samples were collected within $8 \mathrm{~h}$ of post-mortem and consisted of abdominal adipose tissue, lienal artery, bone, yellow bone marrow, red bone marrow, coronary artery, gastric mucosa, joint cartilage, thoracic lymph node, tonsils, bladder, gall bladder, abdominal aorta, thoracic aorta, medulla oblongata and nervus ischiadicus (Table 1). DNA extraction was carried out according to the recommendations of the NucleoSpin Tissue DNA extraction kit manufacturer (Macherey-Nagel, Düren, Germany), with DNA concentrations and quality parameters provided in Supplementary Table 1. Four anonymous genomic DNA samples for replication sequencing were provided by the Estonian Genome Center of the University of Tartu (EGC). Whole mtDNA was amplified in two amplicons $(8,225$ and $9,447 \mathrm{bp}$ ) by PCR using two pairs of primers. PCR amplicons were enzymatically fragmented, prepared as a 48-plex sequencing library and analysed using an Illumina HiSeq2000 instrument with $101 \mathrm{bp}$ paired-end reads according to the manufacturer's protocols. Confirmation of mtDNA position 16,093 heteroplasmic samples and the 300-320 D-loop region in three individuals was performed by Sanger sequencing. The data were analysed using freely available software. All details of methods used are provided in Supplementary Methods.

\section{Results}

A total of 260 million paired-end reads were generated for 16-tissue body panels in three unrelated males (48 samples in total) and for four replicates (8 samples). Each sample library preparation was started from $0.5 \pm 0.1 \mu \mathrm{g}$ of PCR product, but demultiplexing revealed 22 -fold differences between sample raw reads (Supplementary Fig. 1). Mapping and read trimming increased it to 49-fold, but quality score-based filtering and PCR duplicate removal finally reduced sample variability to 25 -fold. The post-filtering mean quality score was 33.5 and $84 \%$ of reads (median) had a quality score of $\geq 30.80 .2 \%$ (median) of reads mapped to mtDNA reference and $41.5 \%$ of reads (median) were removed afterwards during the PCR duplicate filtering step. The average MAF fraction over the full mtDNA 
Table 1 Heteroplasmy overview at position 16,093 of individual SJ600

\begin{tabular}{|c|c|c|c|c|c|c|}
\hline Tissue & Sequencing depth & Major allele & $\mathrm{C}^{\mathrm{a}}$ & Minor allele & $\mathrm{T}^{\mathrm{a}}$ & $\operatorname{MAF}(\%)$ \\
\hline Bladder & 12,339 & $\mathrm{C}$ & 4,691 & $\mathrm{~T}^{\mathrm{b}}$ & 7,645 & 62.0 \\
\hline Lienal artery & 5,343 & $\mathrm{C}$ & 2,625 & $\mathrm{~T}^{\mathrm{b}}$ & 2,717 & 50.9 \\
\hline Coronary artery & 17,265 & $\mathrm{C}$ & 11,123 & $\mathrm{~T}^{\mathrm{b}}$ & 6,139 & 35.6 \\
\hline Thoracic aorta & 6,870 & $\mathrm{C}$ & 4,446 & $\mathrm{~T}^{\mathrm{b}}$ & 2,422 & 35.3 \\
\hline Abdominal aorta & 9,828 & $\mathrm{C}$ & 6,762 & $\mathrm{~T}^{\mathrm{b}}$ & 3,061 & 31.2 \\
\hline Nervus ischiadicus & 8,391 & $\mathrm{C}$ & 6,190 & $\mathrm{~T}^{\mathrm{b}}$ & 2,200 & 26.2 \\
\hline Gall bladder & 5,433 & $\mathrm{C}$ & 4,041 & $\mathrm{~T}^{\mathrm{b}}$ & 1,391 & 25.6 \\
\hline Bone & 11,603 & $\mathrm{C}$ & 9,047 & $\mathrm{~T}^{\mathrm{b}}$ & 2,555 & 22.0 \\
\hline Gastric mucosa & 11,954 & $\mathrm{C}$ & 9,692 & $\mathrm{~T}^{\mathrm{b}}$ & 2,222 & 18.6 \\
\hline Abdominal adipose tissue & 3,724 & $\mathrm{C}$ & 3,039 & $\mathrm{~T}^{\mathrm{b}}$ & 685 & 18.4 \\
\hline Medulla oblongata & 12,985 & $\mathrm{C}$ & 11,266 & $\mathrm{~T}^{\mathrm{b}}$ & 1,717 & 13.2 \\
\hline Thoracic lymph node & 14,600 & $\mathrm{C}$ & 12,672 & $\mathrm{~T}^{\mathrm{b}}$ & 1,926 & 13.2 \\
\hline Tonsils & 10,827 & $\mathrm{C}$ & 9,752 & $\mathrm{~T}^{\mathrm{b}}$ & 1,073 & 9.9 \\
\hline Joint cartilage & 14,444 & $\mathrm{C}$ & 13,074 & $\mathrm{~T}^{\mathrm{b}}$ & 1,368 & 9.5 \\
\hline Yellow bone marrow & 18,223 & $\mathrm{C}$ & 16,607 & $\mathrm{~T}^{\mathrm{b}}$ & 1,613 & 8.9 \\
\hline Red bone marrow & 11,650 & $\mathrm{C}$ & 11,051 & $\mathrm{~T}^{\mathrm{b}}$ & 598 & 5.1 \\
\hline
\end{tabular}

a Allele read count

b The reference allele according to the revised Cambridge reference sequence (rCRS)

sequence is $<0.15 \%$ (Supplementary Table 2). PCR amplification generated unequal coverage over mtDNA, preferring one amplicon to another and bringing out two overlapping regions in amplicons (Fig. 1a). In KA522, the joint cartilage tissue sample was removed as a result of low-sequencing coverage and quality, caused by lower ( $p=0.0003)$ purity of the original DNA sample (Supplementary Table 1$)$.

Forty-seven samples were mapped against a reference sequence to investigate heteroplasmy in mtDNA. Preliminary analysis revealed heteroplasmy with MAF of $<10 \%$ at mtDNA positions $302,309,310$, and 316 in the three studied DNA body panels but Sanger resequencing of poly-C tracks in mtDNA bases 302-310 and 312-317 showed no heteroplasmy in this region, and confirmed only KT538 C insertion at mtDNA position 309 (named C309CC, Supplementary Fig. 2). That demonstrates that MPS mapping output needs Sanger confirmation under these conditions. The significant heteroplasmy (>10\%) was detected only in individual SJ600 at D-loop position 16,093 (m.16093T > C) (Fig. 1b). Red and yellow bone marrow had 5.1 and $8.9 \%$ heteroplasmy, respectively. Abdominal aorta, thoracic aorta, coronary artery and lienal artery clustered as one group and showed heteroplasmy in the range of $31.2-50.9 \%$, while $62.0 \%$ mtDNA heteroplasmy was detected in the bladder (Table 1). Three tissues with variable heteroplasmy of 5-50\% were examined in detail and confirmed by independent Sanger sequencing (Fig. 1c-d). Two other individuals (KT538 and KA522) had no heteroplasmy at position 16,093 .
Four blood DNAs from the EGC were analysed as duplicates to show the assay reproducibility. The median sequencing depth of all eight samples varied from 4,832 to 11,531 and at least $99.5 \%$ of all the nucleotides had $>1,000$ fold sequencing depth (Supplementary Table 3). In total, eight heteroplasmic positions were detected at $>5 \%$ MAF. Heteroplasmic MAF variance between the replicates was $1.5-25.8 \%$ and the correlation coefficients were 0.88-0.99 (Supplementary Fig. 3).

\section{Discussion}

mtDNA is highly represented in somatic cells, making it as a considerable DNA source after nuclear DNA. Nevertheless, the "total length" of mtDNA per cell is up to $20 \mathrm{Mb}$, which is still 400 times less than the amount of diploid nuclear DNA and therefore mtDNA needs specific enrichment before the MPS analysis. Despite the success of capture-probe-based mtDNA enrichment ( $\mathrm{Li}$ et al. 2012), PCR is still the "gold standard" for selection and amplification of small, targeted DNA regions, like mtDNA. In addition, the results indicate that PCR-introduced errors should not be a significant problem as regards this approach (Li et al. 2010; Zaragoza et al. 2010). We used 22 PCR cycles to reduce possible bias accumulation and ensured an efficient PCR yield with $0.2-1.2 \mu \mathrm{g}$ of input DNA. The size-selected library was repaired and amplified using ten additional cycles, which were too intense because $41.5 \%$ of mapped reads were eliminated during PCR duplicate filtering (Supplementary Fig. 1). 

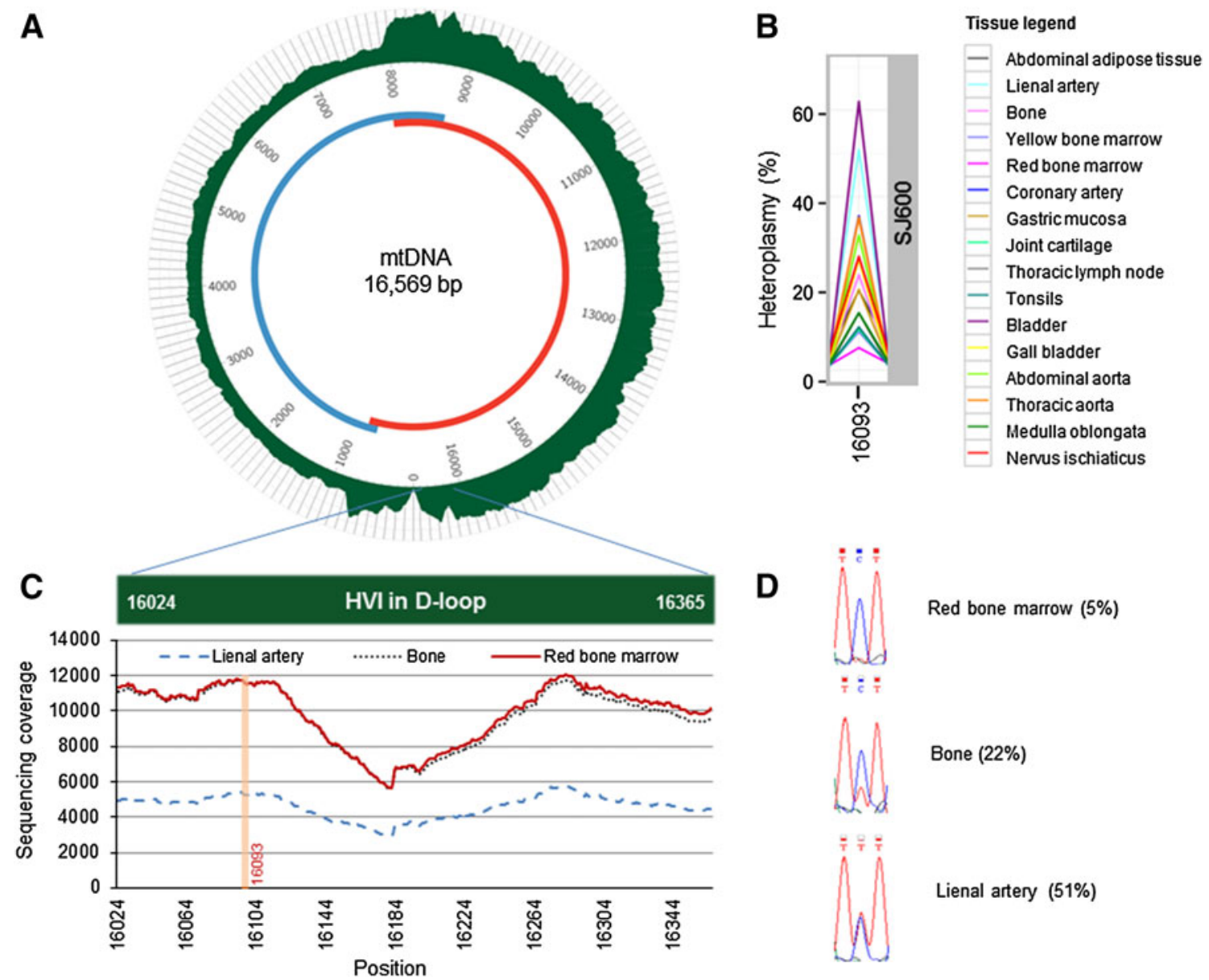

Red bone marrow (5\%)

Bone (22\%)

Lienal artery $(51 \%)$

Fig. 1 Full mtDNA amplification and the confirmation of results. a mtDNA was amplified using two overlapping PCR products, blue and red circles. Average coverage density is shown (green). b Position 16,093 heteroplasmy over the SJ600 body panel. c Sequencing coverage in hypervariable region (HVRI) of D-loop.

The heteroplasmy threshold is an important parameter for accurate detection of heteroplasmy. The threshold calculations based on nuclear DNA control libraries demonstrated that the average proportion of mutations per base was $0.06 \%$ and no base was mutated at greater than $0.8 \%$ frequency (He et al. 2010). A similarly low threshold $(1.3 \%)$ was reported using synthetic DNA fragments with variable allelic ratios (Zhang et al. 2012), providing the acceptable 5-10 \% cut-off for heteroplasmy (Li et al. 2010; Sosa et al. 2012). At the same time, attention should be paid to the fact that artificial quality control DNAs are only useful for assessing indexed library amplification, cluster generation and MPS itself, but are not suitable for sample enrichment and DNA quality issues. In our study, the detected variability at position 16,093 was confirmed by Sanger sequencing at 5, 22 and $51 \%$ MPS-detected heteroplasmy levels. As expected, $5 \%$ heteroplasmy is not detectable by dideoxy-terminator sequencing, $22 \%$ heteroplasmy remains unquantified, and $51 \%$ heteroplasmy
Coverage in the three different tissues of individual SJ600 is shown. d Sanger sequencing confirmation of position 16,093 heteroplasmy. The percentage is from MPS analysis and chromatogram from Sanger sequencing

correlates as a 1:1 polymorphic allelic ratio (Fig. 1d). As our experiment was designed without internal controls to detect the MPS sensitivity threshold, we are not able to report the number of true-positive heteroplasmies without a confirmation experiment. Nevertheless, we detected a great range of position 16,093 heteroplasmy in the human body, contributing to a previous multi-tissue analysis (He et al. 2010). In that previous study, somatic heteroplasmy ranging 7-91\% was found in one of the two study patients. However, the authors studied significantly fewer tissue samples from the same autopsy individual, with little overlap to the panel used in our study. Furthermore, colorectal cancer as the cause of death in (He et al. 2010), could influence the extent of somatic heteroplasmy.

Unfortunately our analysed tissues did not include blood samples, but according to other recent reports reflecting low-level heteroplasmy in blood (Andrew et al. 2011; Avital et al. 2012; Payne et al. 2012), we can speculate that blood (leukocytes) may have lower heteroplasmy level 
than the rest of human body. Because red bone marrow consists mainly of hematopoietic tissue giving rise to all blood cell types, we used red bone marrow (heteroplasmy $5.1 \%$ ) as a possible alternative to blood in our study. Also, it is suggested that rapidly dividing tissues such as blood cells or bone marrow may have the ability to eliminate cells with mtDNA mutations during life, whereas "postmitotic" tissues that no longer divide do not eliminate these cells and may instead favour accumulation of mutant mtDNA genomes. Hence, this could clarify why some inherited mtDNA mutations can be lost from the blood but be detected in other post-mitotic tissues (Payne et al. 2012). Our findings are in line with this, demonstrating very low frequency of heteroplasmy in rapidly dividing red and yellow bone marrow tissues, with 5 and $9 \%$ of heteroplasmy, respectively.

According to our findings, position 16,093 was heteroplasmic in all 16 studied tissues in one individual, varying from $5.1 \%$ in red bone marrow to $62.0 \%$ in the bladder. The differences between organ groups can be explained by random segregation of mtDNA during oogenesis and in somatic tissue development. It is now believed that somatic segregation may lead to high levels of mutant mtDNA in some progenitor cells and low levels in others. In addition, due to the high copy number of mtDNAs, a mutation in some of the mtDNA results in a mixture of variant mitochondrial genomes within a mitochondrion, cell, tissue, organ, or individual. However, it is impossible to predict the cell types or tissues to which mutant molecules will segregate during the ontogenesis. It is likely that such heteroplasmic variability in our study is a result of random genetic drift and not strongly associated with certain developmental stages or tissue differentiation processes. These findings could explain the unequal distribution of the different genotypes, not only between the three germinal layers (endoderm, mesoderm and ectoderm), but also between derivatives of the same germinal layer. In our panel that mostly contains tissues of mesodermal origin, heteroplasmy at position 16,093 differed by more than $40 \%$ between two mesodermal derivatives-bone marrow and lienal artery. In contrast, the same heteroplasmic frequency $(9 \%)$ was present in tissues of different origin, such as tonsils and joint cartilage, which are of endodermal and mesodermal origin, respectively. Interestingly, our results clearly demonstrated similar high-heteroplasmic frequency in various blood vessels-abdominal aorta, thoracic aorta, coronary artery and lienal artery, ranging $31-51 \%$. Arteries carry oxygenated blood away from the heart to the tissues that require oxygen. These findings may partially be interpreted by the production of oxygen radicals during oxidative phosphorylation and common mesodermal origin. The fact that in bladder tissue we observed the highest frequency of heteroplasmy could be explained by its tissue-specific prevalence, as also indicated by the high incidence of mtDNA mutations in bladder cancer (Guney et al. 2012).

In conclusion, our results demonstrate that screening of a tissue panel using MPS technology provides surprising data that reveals somatic heteroplasmy even among the limited number of study subjects. Our findings give clear reason to speculate that mtDNA heteroplasmic frequency, distribution, and even its possible role in complex diseases or phenotypes could be severely underestimated, evoking the need for more thorough tissue-based screenings at different age groups to provide more comprehensive understanding about its biological and clinical importance.

Acknowledgments The authors would like to thank pathologist Dr Enn Jõeste, Ms Heesi Annus and Dr Tambet Teesalu for providing samples, and Dr Lili Milani and Prof Andres Metspalu for performing sequencing in the EGC. We also thank Priit Palta, Tarmo Puurand and Mart Kals for their contribution to the analysis and discussion. This work was supported by targeted financing from the Estonian Government (SFO180142s08), grant SF0180044s09 from the Estonian Ministry of Education and Research, the FP7 grant OPENGENE, and by Enterprise Estonia grant EU30020.

Open Access This article is distributed under the terms of the Creative Commons Attribution License which permits any use, distribution, and reproduction in any medium, provided the original author(s) and the source are credited.

\section{References}

Andrew T, Calloway CD, Stuart S, Lee SH, Gill R, Clement G, Chowienczyk P, Spector TD, Valdes AM (2011) A twin study of mitochondrial DNA polymorphisms shows that heteroplasmy at multiple sites is associated with mtDNA variant 16093 but not with zygosity. PLoS ONE 6(8):e22332

Avital G, Buchshtav M, Zhidkov I, Tuval Feder J, Dadon S, Rubin E, Glass D, Spector TD, Mishmar D (2012) Mitochondrial DNA heteroplasmy in diabetes and normal adults: role of acquired and inherited mutational patterns in twins. Hum Mol Genet 1(19):4214-4224

Goto H, Dickins B, Afgan E, Paul IM, Taylor J, Makova KD, Nekrutenko A (2011) Dynamics of mitochondrial heteroplasmy in three families investigated via a repeatable re-sequencing study. Genome Biol 12(6):R59

Guney AI, Ergec DS, Tavukcu HH, Koc G, Kirac D, Ulucan K, Javadova D, Turkeri L (2012) Detection of mitochondrial DNA mutations in nonmuscle invasive bladder cancer. Genet Test Mol Biomark 16(7):672-678

He Y, Wu J, Dressman DC, Iacobuzio-Donahue C, Markowitz SD, Velculescu VE, Diaz LA Jr, Kinzler KW, Vogelstein B, Papadopoulos N (2010) Heteroplasmic mitochondrial DNA mutations in normal and tumour cells. Nature 464(7288): 610-614

Li M, Schonberg A, Schaefer M, Schroeder R, Nasidze I, Stoneking M (2010) Detecting heteroplasmy from high-throughput sequencing of complete human mitochondrial DNA genomes. Am J Hum Genet 87(2):237-249 
Li M, Schroeder R, Ko A, Stoneking M (2012) Fidelity of captureenrichment for mtDNA genome sequencing: influence of NUMTs. Nucleic Acids Res 40(18):e137. doi:110.1093/nar/gks1499

Payne BA, Wilson IJ, Yu-Wai-Man P, Coxhead J, Deehan D, Horvath R, Taylor RW, Samuels DC, Santibanez-Koref M, Chinnery PF (2012) Universal heteroplasmy of human mitochondrial DNA. Hum Mol Genet 22(2):384-390

Sosa MX, Sivakumar IK, Maragh S, Veeramachaneni V, Hariharan R, Parulekar M, Fredrikson KM, Harkins TT, Lin J, Feldman AB, Tata P, Ehret GB, Chakravarti A (2012) Next-generation sequencing of human mitochondrial reference genomes uncovers high heteroplasmy frequency. PLoS Comput Biol 8(10):e100 2737

Zaragoza MV, Fass J, Diegoli M, Lin D, Arbustini E (2010) Mitochondrial DNA variant discovery and evaluation in human Cardiomyopathies through next-generation sequencing. PLoS ONE 5(8): 12295

Zhang W, Cui H, Wong LJ (2012) Comprehensive one-step molecular analyses of mitochondrial genome by massively parallel sequencing. Clin Chem 58(9):1322-1331 